\title{
Computational Image Analysis of Guided Acoustic Waves Enables Rheological Assessment of Sub-nanoliter Volumes
}

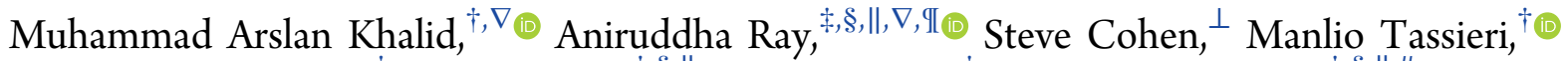
Andriejus Demčenko, ${ }^{\dagger}$ Derek Tseng, ${ }^{\ddagger}, \S, \|$ Julien Reboud, ${ }^{\dagger \odot}$ Aydogan Ozcan, ${ }^{*},,,,, \|, \# \odot$ and Jonathan M. Cooper* ${ }^{*} \dagger$

${ }^{\dagger}$ Division of Biomedical Engineering, School of Engineering, University of Glasgow, Glasgow G12 8LT, United Kingdom ${ }^{\ddagger}$ Electrical and Computer Engineering Department, ${ }^{\S}$ Bioengineering Department, "California Nano Systems Institute (CNSI), ${ }^{\perp}$ Neuroscience, and ${ }^{\#}$ Department of Surgery, David Geffen School of Medicine, University of California, Los Angeles (UCLA), California, United States

\section{Supporting Information}

ABSTRACT: We present a method for the computational image analysis of high frequency guided sound waves based upon the measurement of optical interference fringes, produced at the air interface of a thin film of liquid. These acoustic actuations induce an affine deformation of the liquid, creating a lensing effect that can be readily observed using a simple imaging system. We exploit this effect to measure and analyze the spatiotemporal behavior of the thin liquid film as the acoustic wave interacts with it. We also show that, by investigating the dynamics of the
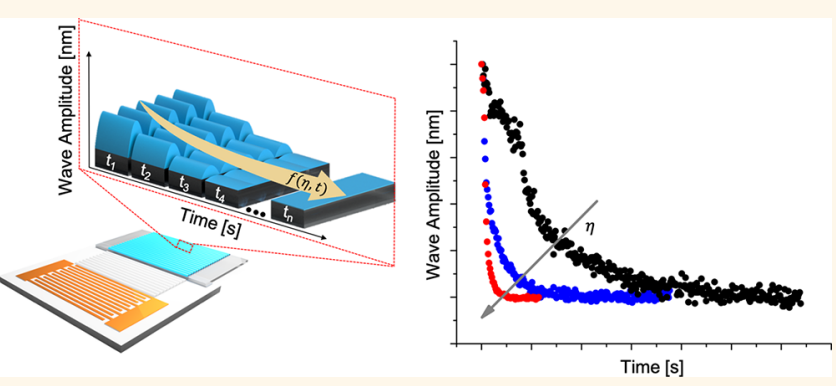
relaxation processes of these deformations when actuation ceases, we are able to determine the liquid's viscosity using just a lens-free imaging system and a simple disposable biochip. Contrary to all other acoustic-based techniques in rheology, our measurements do not require monitoring of the wave parameters to obtain quantitative values for fluid viscosities, for sample volumes as low as $200 \mathrm{pL}$. We envisage that the proposed methods could enable high throughput, chip-based, reagent-free rheological studies within very small samples.

KEYWORDS: computational image analysis, holography, guided acoustic waves, microscopy, rheology

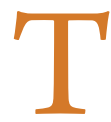
he visualization and characterization of acoustic waves as they propagate in media have previously been used to elucidate material properties and gain a deeper understanding of physical phenomena, including the RamanNath effect and Brillouin scattering. ${ }^{1,2}$ For example, it has previously been shown that wave propagation through solid media can reveal valuable information about the mechanical properties of materials ${ }^{3}$ such as local stresses, densities, and elastic moduli. ${ }^{4}$ In the case of the study of the propagation of acoustic waves in liquids, as they pass either through the bulk or across boundary interfaces and discontinuities, it is possible to measure local viscosities, thermal conductivities, and thermoelastic relaxation processes. ${ }^{5}$ In this context, it is already well established that monitoring liquids' viscosities (for Newtonian fluids) or viscoelasticities (for non-Newtonian fluids) is of importance in industry, for example, in the formulation of paint and processed food as well as in biomedical applications such as measuring blood viscosity. ${ }^{6}$
Conventional bulk rheology measurements are usually performed by means of large benchtop viscometers and rheometers (often requiring several milliliters of sample). Recently, microrheology, ${ }^{7,8}$ techniques requiring only a few tens of microliters of sample have emerged, although many of these protocols require complex sample processing, including the addition of labels or tracer particles. ${ }^{9}$ Other methods have also been reported that use nanoliter sample volumes, although these all require auxiliary equipment such as benchtop optical lasers, ${ }^{10}$ microscopes ${ }^{11}$ (e.g., atomic force microscopy), ${ }^{12}$ nanoliter droplet dispensers, ${ }^{12}$ or complex microfluidic channel designs, ${ }^{11}$ making them cumbersome and unsuitable as portable instruments.

Received: April 26, 2019

Accepted: September 6, 2019

Published: September 6, 2019 
Guided acoustic waves have previously been used in rheological applications ${ }^{13,14}$ by measuring their attenuation. Such mechanical excitations, including those using surface acoustic waves (SAWs) and Lamb-type waves, have also been used to drive liquid actuation in microfluidic systems. ${ }^{15-18}$ In this work, we make use of the capability of acoustic waves to deform a subnanoliter-scale liquid volume and monitor the dynamics of its relaxation when the actuation is turned off (i.e., in contrast with all previous rheological measurement techniques there are no acoustic waves propagating in the liquid during the measurement).

Different methods have previously been implemented for gathering a better understanding of wave propagation. For instance, Schlieren imaging and interferometric systems such as laser Doppler vibrometers (LDVs) have been used for studying acoustic wave propagation and visualization of acousto-optic interactions, ${ }^{19}$ where light is modulated by ultrasonic waves to generate Fraunhofer diffraction patterns. ${ }^{3}$ However, optical aberrations (e.g., in Schlieren visualization) can often result in reduced contrast and poor fidelity, ${ }^{19}$ whereas in the case of LDV strong acousto-optic interaction in condensed medium can result in large measurement errors, especially when applied to liquids. ${ }^{20}$ Alternative methods using holography have also been used to visualize $\sim 1 \mathrm{MHz}$ waves but these require the addition of reagents, acting as reporter particles to generate the holograms. ${ }^{21}$ Recently, a fast but low-resolution method for visualizing acoustic beams was proposed, ${ }^{22}$ where an excited region within a thin liquid layer produced an optical pattern because of the local deformation of the liquid film.

Here, we present a simple optical method for imaging small amplitude guided waves of wavelength $\lambda$ in plates coated by a thin fluid layer of thickness $2 a$ (with $2 a \ll \lambda$ ) and demonstrate its application for measuring the viscosity of liquid samples with subnanoliter volumes, without monitoring wave parameters. Using computational imaging ${ }^{23,24}$ of these guided waves at ultrasonic frequencies $(\sim 10 \mathrm{MHz})$, we demonstrate their visualization over a wide field-of-view $\left(\sim 30 \mathrm{~mm}^{2}\right)$ within a lens-free system. The method was validated by a direct visualization of Lamb-type waves and is supported by analytical and numerical models.

In our configuration, the liquid-air interface was first deformed using acoustic actuation and the relaxation dynamics of the deformation was monitored, once the acoustic excitation was switched off. As stated, contrary to all previous techniques, no acoustic wave is propagating when the measurement is carried out. We corroborate this by measuring the rheological properties of aqueous mixtures of glycerol and polyethylene glycol, $M_{\mathrm{r}}=400$ (PEG400). We demonstrated the ability of the technique to investigate nanoliter-scale volumes of liquids (where other approaches require orders of magnitude larger sample volumes). Our approach does not require the addition of reagents or labels and is contactless (these being "ideal" requirements in biological studies). We envisage that in the future the device can not only be integrated with microfluidics and lab-on-chip platforms for high-throughput characterization of bioliquids but could also be used for other applications such as inspection of materials properties (e.g., industrial wafer stress-testing or rapidly investigating wafer defects by exploring deformations in a thin film of liquid).

\section{RESULTS AND DISCUSSION}

Imaging System. The imaging system comprised a threedimensional (3D) printed housing to hold an array of green light-emitting diodes (LED) that can be selectively illuminated and controlled using a microcontroller. The imaging system also included an optical band-pass filter (532 nm), a 3D printed sample holder to align a disposable waveguide with the piezoelectric interdigitated transducer (IDT) and a complementary metal oxide semiconductor (CMOS) sensor with a pixel pitch of $1.67 \mu \mathrm{m}$, Figure 1 . (a)

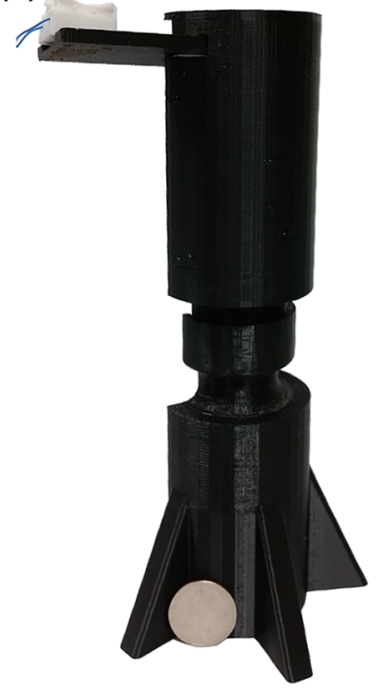

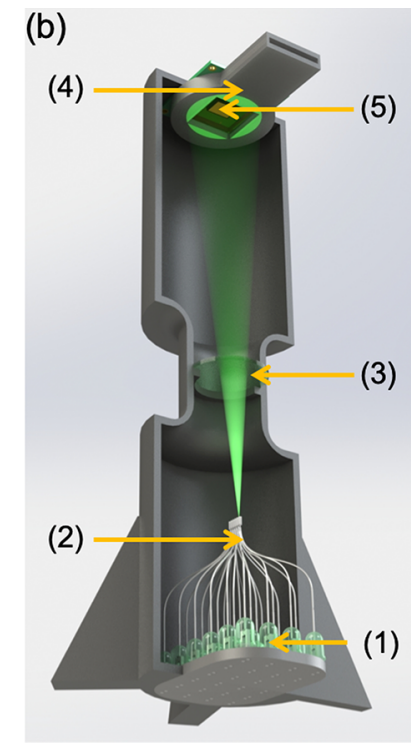

Figure 1. Three-dimensional printed, acoustic computational imaging system. (a) The lens-free computational imaging system integrated with an ultrasonic transducer. (b) The schematic of the device. The device contains 20 LEDs (1) with a selectable switch using an onboard microcontroller. The light from each LED was coupled into an optical fiber (2) that passes through a narrow band filter (3). The sample, illuminated from the bottom, was placed on the lens-free microscope using a detachable tray containing the SAW IDT (4). The CMOS imager (5) was placed directly on top of the sample $(\sim 1 \mathrm{~mm}$ high $)$ to record the transmitted light from the sample.

The 20 fiber-coupled LED array was used to illuminate the sample from below, ${ }^{25}$ as shown in Figure 1. It was designed to generate a set of subpixel shifted images, which were then used to digitally synthesize images with subpixel resolution. ${ }^{26-28}$ The disposable biochip was coupled to the IDT using a thin layer of polyethylene glycol (PEG 400) which we found to be a stable coupling agent. The transmitted light was recorded by the CMOS sensor, which was placed at $\sim 1 \mathrm{~mm}$ distance from the sample, so that the sample field-of-view was equal to the active area of the CMOS imager. A Rayleigh wave, generated on the lithium niobate $\left(\mathrm{LiNbO}_{3}\right)$ transducer, coupled into the platelike glass biochip, with finite dimensions, creating a standing Lamb-type wave, Figure 2.

This standing wave induced an affine deformation of the thin liquid layer on the substrate of thickness $2 h$ (with $2 a \ll$ $2 h$ ), which was detected using the CMOS image sensor to measure the bright and dark fringes generated by the distortion of the optical wavefronts while passing though the liquid-air interface, Figure 3.

We investigated how the liquid layer deformation, at its maximum at the standing wave antinodes and at its minimum at the standing wave nodes, could be used to create nanolensing effects. The induced deformation of the liquid's 


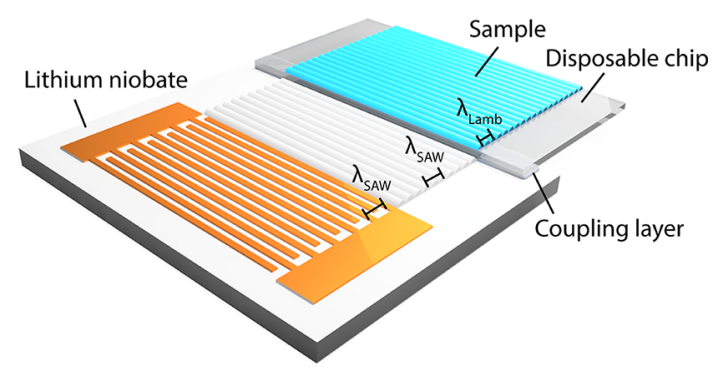

Figure 2. A schematic of an IDT on a lithium niobate $\left(\mathrm{LiNbO}_{3}\right)$ wafer. The pitch of the IDT was the same as the wavelength $\left(\lambda_{\mathrm{SAW}}\right)$ of the Rayleigh wave. The Rayleigh wave couples into disposable (glass) biochip as a Lamb-type wave. The IDT and the disposable biochip are coupled using a thin layer of PEG400.

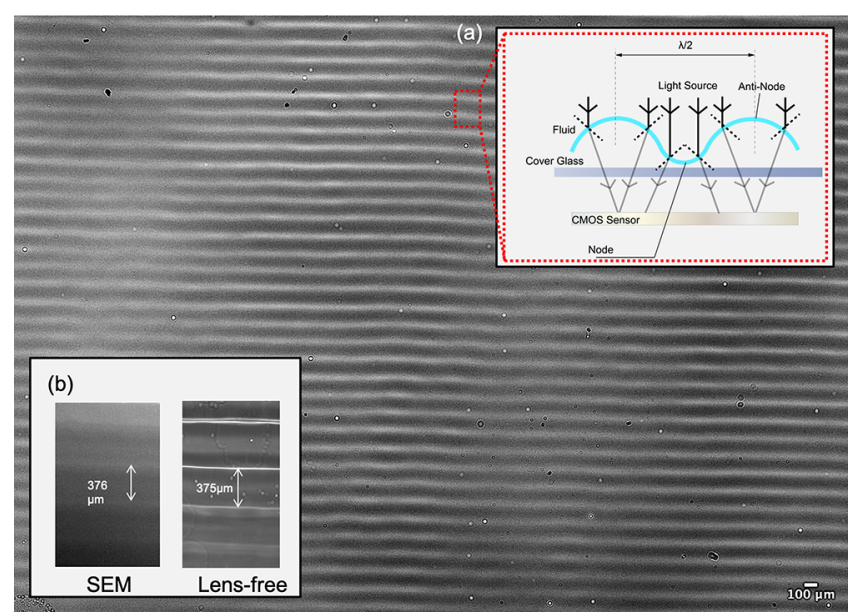

Figure 3. Lamb-type wave imaging. (a) An image of the Lamb-type wave in a thin film of glycerol $(60 \% \mathrm{w} / \mathrm{w}$ in water) on a glass superstrate actuated by a $9.71 \mathrm{MHz}$ SAW transducer. The SAW beam couples into the superstrate as a Lamb wave and reflects back from the glass-air boundary to create a standing wave in the liquid. The red-dotted box shows the cartoon of the underlying principle. At the nodes of the standing wave, the surface displacement was minimum, whereas at the antinodes the surface displacement was maximum creating a lens-like profile in the liquid. The incident light was defocused and focused respectively and was collected at the CMOS sensor in the form of dark and bright fringes allowing imaging of the wave. (b) Comparison between an SEM image and a lens-free image of a UV curable polymer used to create permanent wave patterns. Full frames of the images are available in Supporting Information Video S1.

surface generates an optical profile that can be modeled to a first approximation as a sinusoidal function, allowing an estimation of the deformation height using a ray-tracing model (calculated as about $150 \mathrm{~nm}$, Figure 4). As a result, the light passing through the liquid-air interface can be both focused (bright fringes) and defocused (dark fringes), Figure 4b,c.

The corrugation height of the deformations in the SAW actuation of the liquid surface at the interface depends upon the frequency and amplitude of the standing wave as well as on the liquid's physical properties. ${ }^{29-32}$ Our lens-free optical imaging configuration has unit-magnification. ${ }^{33}$ To quantitatively analyze the intensity of the fringes' patterns, we developed a graphic user interface that allowed us to load a sequence of images (or a video) to analyze selected regions of interest, frame by frame. The wavelength of the periodic deformation of the liquid was calculated using a fast Fourier (a)

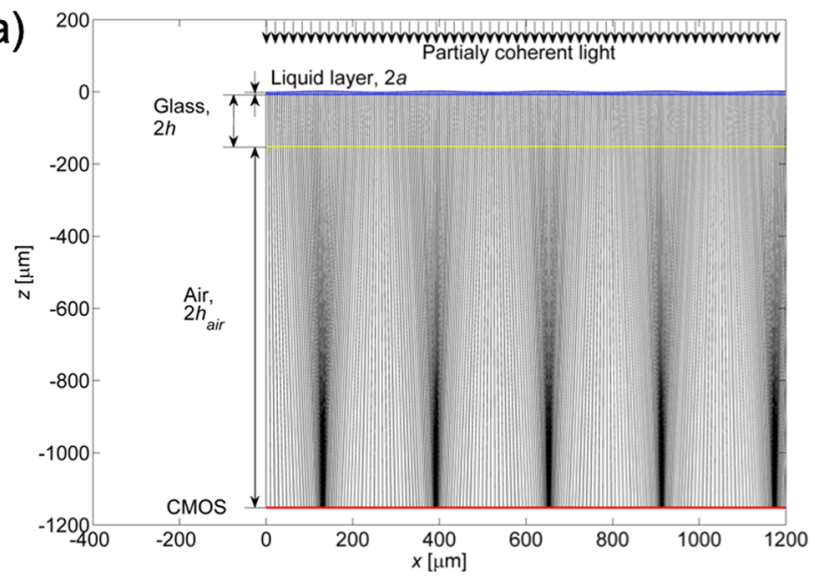

(b)
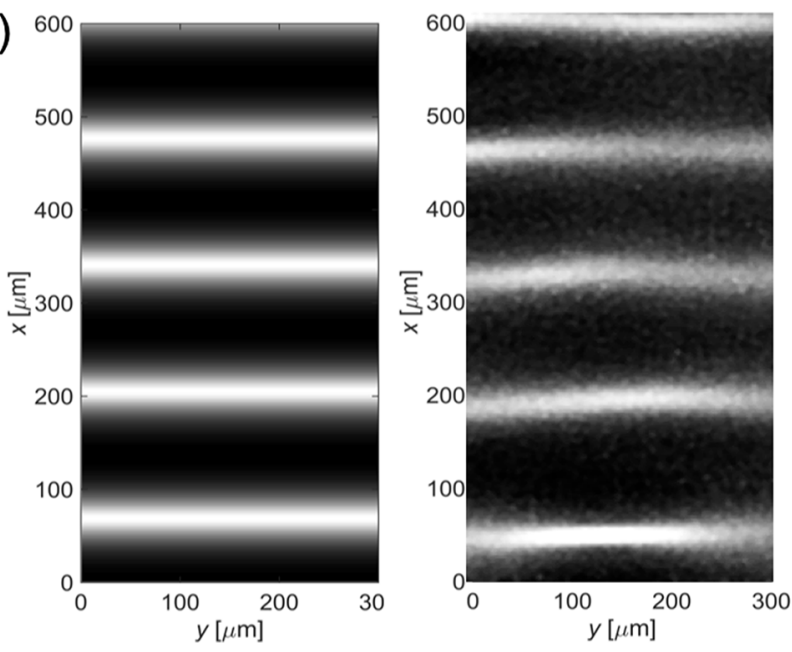

Figure 4. Liquid deformations and optical elements. (a) Raytracing model geometry for the lens-free imaging system. The light rays from a partially coherent light source pass through a liquid layer of thickness $2 a$ under a sinusoidal corrugation of the liquid surface. The light refracts as it passes through different media, that is, the liquid (mineral oil), glass, and air before being detected by the CMOS sensor. The corrugation height of the liquid increases with the excitation amplitude. (b) Modeled liquid deformation for mineral oil sample. The dark area represents the pressure nodes in the glass plate where the liquid deformation was minimum, whereas the bright regions represents the antinodes. (c) Corresponding image for experimental measurement. The modeling shows that the corrugation height is $0.154 \mu \mathrm{m}$.

transform (FFT) algorithm. The temporal response of the deformation was calculated by implementing a dynamic spline fitting algorithm as shown in Figure 5.

The results from the lens-free imaging system were validated by creating a steady-state standing acoustic sinusoidal wave pattern by ultraviolet (UV) illumination of a photocurable polymer. This technique enabled us to freeze the induced deformation of the polymer thin layer, showing the spatial periodicity of the elastic waves. This induced structure was visualized by both the lens-free imaging system and a scanning electron microscope (SEM), Figure $3 \mathrm{~b}$. From these images, we estimated the steady-state wavelength at an excitation of 9.71 $\mathrm{MHz}$ to be $375 \mu \mathrm{m}$ in the lens-free image validated by the SEM image, respectively. In the case of liquid films, the effectiveness of the lens-free imaging system was further corroborated using laser Doppler vibrometry. For example, 

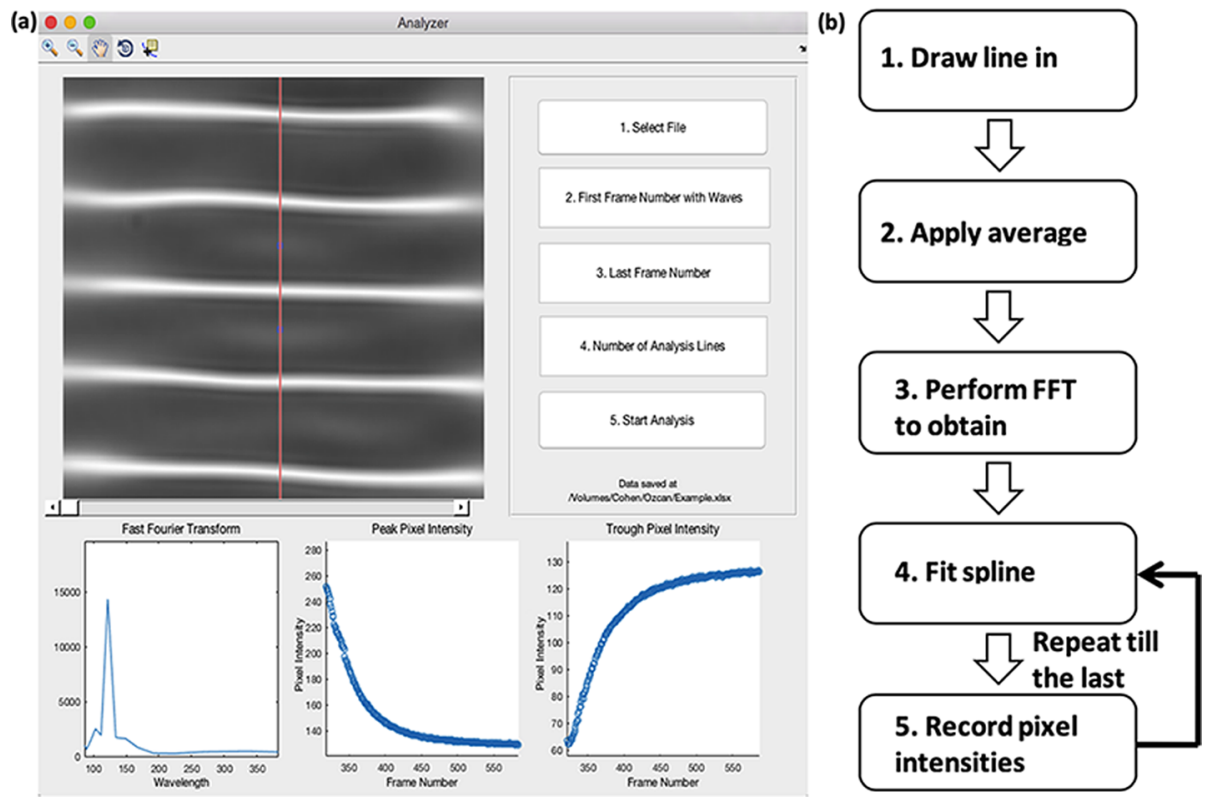

Figure 5. Graphical user interface (GUI). (a) The GUI to measure the wavelength and analyze the transient response. The GUI shows the frame-by-frame preview of the video and allows the user to select the appropriate frame range for further analysis. (b) Process flow of the image processing algorithm. For measuring the phase velocity, a 1D FFT is applied to the region of interest and the output is shown in the GUI. For transient analysis, the region of interest (user drawn lines) is filtered to remove background noise and the normalized pixel intensity is computed. The maximum and minimum intensity values were calculated by fitting a spline curve and the temporal response was plotted in the GUI. Upon selecting a frame, reporting lines were added to analyze multiple regions. The display outputs are (bottom left) the calculated wavelength, (bottom center) the temporal behavior of the image brightness (i.e., relaxation of the antinode) as the SAW is switched off, and (bottom right) the recovery of the dark regions (i.e., the nodes). The temporal response was calculated by implementing a dynamic spline-fitting algorithm.

(a)

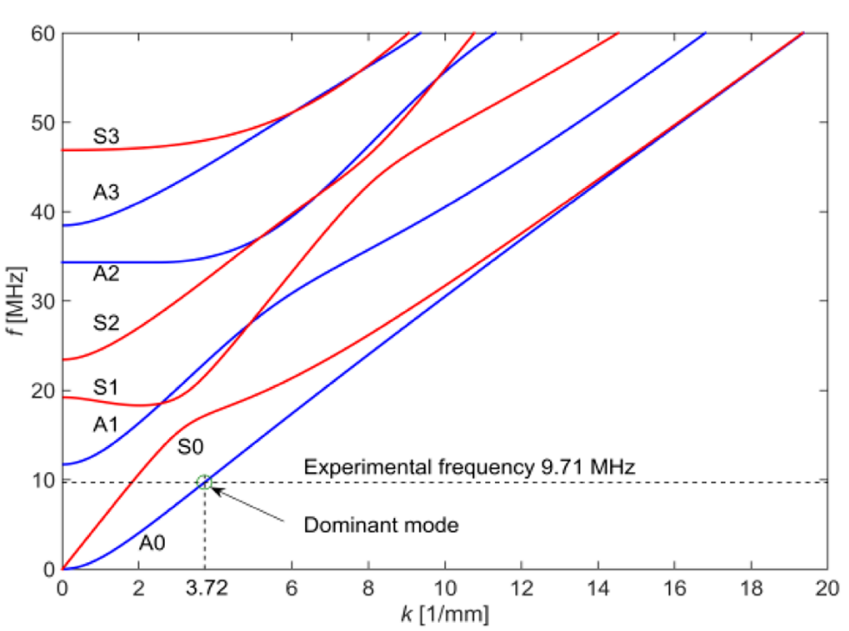

(b)

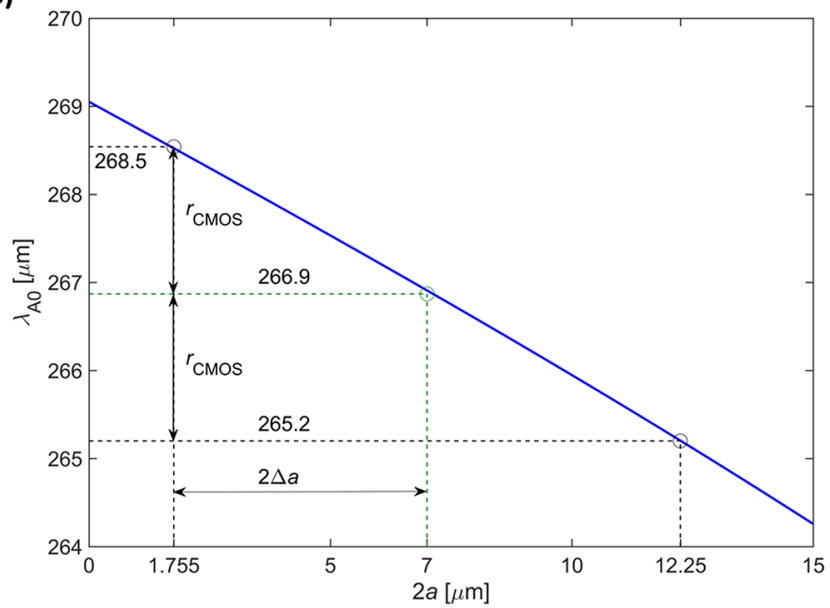

Figure 6. (a) Lamb wave dispersion curves in a glass (biochip) plate of $145 \mu \mathrm{m}$ thickness, where the blue and red colors indicate antisymmetric and symmetric Lamb wave modes, respectively. The green circle marks the experimentally excited $A 0$ Lamb wave mode at 9.71 MHz. (b) Dispersion of antisymmetric guided wave mode versus liquid thickness $2 a$ at $9.71 \mathrm{MHz}$, where blue color shows the analytical result (eq 1). The CMOS sensor resolution $r_{\mathrm{CMOS}}$ shows that the liquid thickness variations $2 \Delta a<5 \mu \mathrm{m}$ can be neglected in the measurement system.

when measuring phase velocity there is no significant difference between the vibrometer $(2534.9 \pm 1.8 \mathrm{~m} / \mathrm{s})$, the lens-free system $(2503.7 \pm 21.6 \mathrm{~m} / \mathrm{s})$ and the analytical value $(2525.9 \mathrm{~m} / \mathrm{s})$.

Theoretical Analysis. We considered the analysis of small amplitude ultrasonic guided waves (with a wavenumber $k=$ $2 \pi f / c$, where $f$ is the frequency, $c$ is the guided wave phase velocity) propagating in a nonviscous liquid-solid bilayer. The viscosity of the liquid was disregarded because its effect on the phase velocity of Lamb waves was negligible compared to that on their attenuation. ${ }^{34}$ The liquid layer (thickness, $2 a$ ) was characterized using the ultrasonic wave phase velocity in the liquid, $c_{\mathrm{F}}$, and the volumetric mass density, $\rho_{\mathrm{F}}$. The elastic solid layer (thickness, $2 h$ ) and volumetric mass density $\rho$ enables propagation of longitudinal and transversal ultrasonic waves with velocities $c_{\mathrm{L}}$ and $c_{\mathrm{T}}$, respectively. The guided waves in the liquid-solid bilayer were analyzed using the following dispersion equation ${ }^{35}$ 


$$
\begin{aligned}
& 16 r A S \cos \left(2 \frac{a}{h} r\right)+\pi^{2} \frac{\rho_{\mathrm{F}}}{\rho} p^{4}\left(d^{2} \cos (2 p) \sin (2 q)+4 \xi^{2} p q \sin (2 p) \cos (2 q)\right) \\
& \sin \left(2 \frac{a}{h} r\right)=0
\end{aligned}
$$

where

$$
\begin{aligned}
& A=d^{2} \sin (p) \cos (q)+4 \xi^{2} p q \cos (p) \sin (q) \\
& S=d^{2} \cos (p) \sin (q)+4 \xi^{2} p q \sin (p) \cos (q)
\end{aligned}
$$

Equations 2 and 3 represent the dispersion relations for the antisymmetric and symmetric guided modes (Lamb-type wave) in the free solid plate. The remaining coefficients are given by

$$
\begin{aligned}
& p=2 \pi f h \sqrt{\frac{1}{c_{\mathrm{L}}^{2}}-\frac{1}{c^{2}}} \\
& q=2 \pi f h \sqrt{\frac{1}{c_{\mathrm{T}}^{2}}-\frac{1}{c^{2}}} \\
& r=2 \pi f h \sqrt{\frac{1}{c_{\mathrm{F}}^{2}}-\frac{1}{c^{2}}} \\
& d=8 \pi f^{2} h^{2}\left(\frac{1}{c_{T}^{2}}-\frac{2}{c^{2}}\right)
\end{aligned}
$$

where $\omega=4 f h / c_{\mathrm{T}}$ and $\xi=4 f h / c$.

In absence of a liquid layer (i.e., when its thickness is 0 ), eq 1 enabled us to calculate the dispersion curves of the Lamb waves in the free solid plate, Figure 6 (model parameters are listed in Table S1). A cutoff frequency of the higher order Lamb wave mode A1 was at $11.7 \mathrm{MHz}$. Numerical results showed that only the fundamental Lamb wave modes A0 and S0 can exist in the free solid plate below such frequency. The antisymmetric excitation source ensures that the antisymmetric mode $\mathrm{A} 0$ is dominant in the thin glass plate. When the plate was coated by a thin liquid film, the Lamb waves did not couple into the coating liquid (as the phase velocity changes were negligible) but the waves were attenuated by the viscosity of the liquid. ${ }^{34}$ We measured the thickness of the liquid layer at about $7 \mu \mathrm{m}$ with optical microscopy. Our analysis showed that in the cases of thin layers (i.e., $2 a<15 \mu \mathrm{m}$ ), the wavelength of the guided wave of A0 decreases from $\lambda=269.1 \mu \mathrm{m}$ to $\lambda=$ $264.3 \mu \mathrm{m}$. Given the finite resolution of the CMOS sensor, we would not be able to detect phase velocity changes of the guided wave for variations of the liquid film thickness below 5 $\mu \mathrm{m}$ (Figure 6b). Finally, the theoretical framework introduced above indicated that small volumes of liquid (subnanoliters), such as those used in this work, do not influence the wave propagation, confirming the analytical results.

We also show that guided waves with a longer wavelength than the liquid thickness (i.e., $\lambda \gg 2 a$ ) do not couple into the liquid from the glass plate. Because the wave amplitude is much smaller than the liquid thickness $(2 a)$, any possible nonlinear effects such as acoustic streaming, cavitation, jetting, and nebulization of the liquid are either negligible or not possible.

Transient Response and Rheological Application. Investigations of dynamics of standing waves at the liquidair interface can be dated to the pioneering studies of Faraday in $1831,{ }^{36}$ where the formation of standing waves on the free surface of a liquid, subjected to vertical sinusoidal oscillation, was reported. Since then, several attempts have been made $^{37-40}$ to provide a general solution by exploring increasingly complex systems, including inviscid liquids (where the damping effects are neglected), ${ }^{41}$ simple Newtonian liquids ${ }^{42}$ (with a time-invariant viscosity), and generic viscoelastic liquids ${ }^{32}$ (with a frequency-dependent viscosity). In the case of Newtonian liquids, the general analytical approach is often based on solving a Mathieu equation, which describes the forcing of a simple harmonic oscillator by periodic variation of its proper frequency. ${ }^{43}$ The displacement $x(t)$ of a harmonic oscillator can be described by ${ }^{31}$

$$
\begin{aligned}
& \frac{\mathrm{d}^{2} x}{\mathrm{~d} t^{2}}+2 \mu \frac{\mathrm{d} x}{\mathrm{~d} t}+\omega_{0}^{2}(t) x=0 \\
& \omega_{0}^{2}(t)=\omega_{0}^{2}[1+\varepsilon \cos (t)]
\end{aligned}
$$

where $\mu$ is the damping rate, $\varepsilon$ is the amplitude of the force at frequency $\omega$, and $t$ is the time. An analysis of the Mathieu equation shows that the $\varepsilon-\omega$ parameter plane is divided into regions where the displacement $x(t)$ goes to zero at long times (assuming finite damping), and regions (known as resonance tongues) where it grows exponentially without bound. ${ }^{31}$

A thin liquid film was deposited onto the biochip and wide area imaging of Lamb-type waves was performed. The liquid, placed on such a solid substrate, could be assumed to be subjected to an affine sinusoidal deformation, whose amplitude $(\sim 0.154 \mu \mathrm{m})$ was much smaller than the liquid thickness $(\sim 7$ $\mu \mathrm{m})$, with the liquid-air interface mirroring the deformation induced by the guided wave at the solid-liquid interface, as shown in Figure 4. Because the liquid thickness was much smaller than the guided wave's wavelength and as the acoustic wave did not couple into the liquid, it follows that under continuous ultrasonic excitation the deformation of the liquid's surface reached a steady-state amplitude.

Upon switching the ultrasonic actuation off, the acoustic wave energy at the solid-liquid boundary dissipated almost instantaneously (that is, $\sim \mu \mathrm{s}$ ), while the deformation of the liquid at the liquid-air interface relaxed at a much slower rate (i.e., over a few seconds). As stated, the initial deformation (at the point where the acoustic actuation is turned off) mirrored the Lamb-type wave deformations of the surface, as a singlemode pattern. The relaxation processes were analyzed as a relaxation phenomena of a single-mode small instability perturbing an overdamped harmonic oscillator. ${ }^{43-45}$ For such a system, we note that the additional approximation, neglecting the acceleration due to gravity, enables eq 8 to be solved to give a wave amplitude $\left(A_{\kappa}(t)\right)$ that is expected to decrease exponentially

$$
A_{\kappa}(t) \propto \exp (-\zeta t)
$$

with decay rate:

$$
\zeta \propto \frac{\nu}{2 \sqrt{\frac{\sigma \kappa^{3}}{\rho_{\mathrm{F}}}}}
$$

where $\nu$ is the kinetic viscosity of the liquid, $\sigma$ is the liquid-air surface tension, and $\kappa$ is the wavelength of the initial instability. $^{46}$

The transient relaxation responses of different waterglycerol mixtures (differing by less than $10 \%$ in surface tension, but orders of magnitude in viscosity), as well as PEG400-water mixtures, were investigated when the excitation 
of the elastic wave was removed, Figure 7 . The results show that the attenuation rate was faster and the overall relaxation

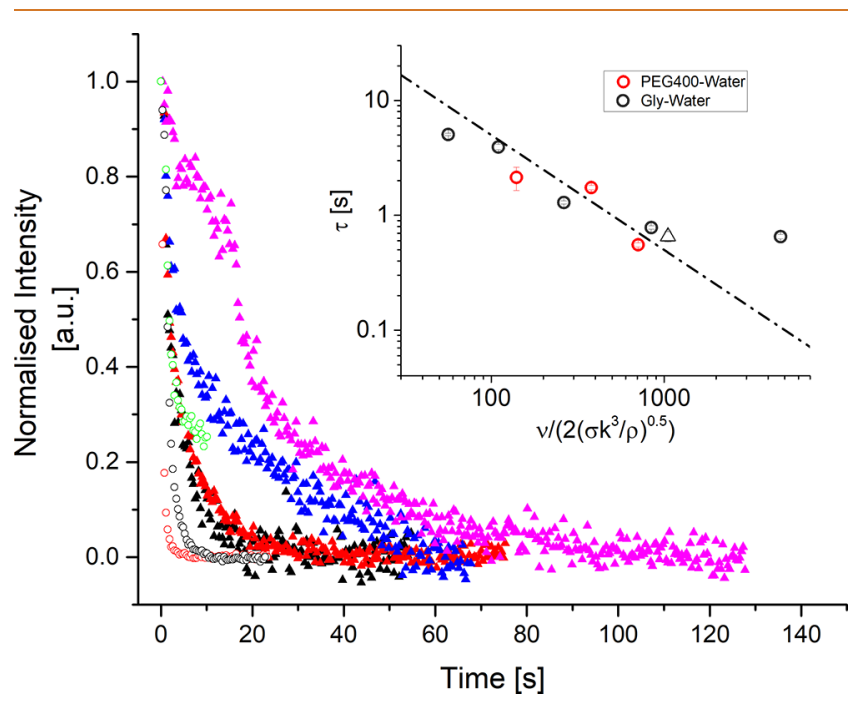

Figure 7. Viscosity measurements. The transient response upon cessation of acoustic actuation of the induced affine deformation of the liquid-air interface of various glycerol-water (triangles) and PEG400-water (circles) mixtures. Measurements were performed as described in Figure 5. The inset shows the existence of an inverse proportionality between the measured time of relaxation and the fluids' viscosity.

time was shorter for more viscous liquids (inset). The measurements agreed with conventional bulk rheology values, Figure S2. When subjected to SAWs, highly viscous liquids (e.g., 100\% glycerol) undergo rapid heating. Figure S3 shows the changes in viscosity induced by the heat generated after $5 \mathrm{~s}$ SAW actuation, consistent with results reported by Zha et al. ${ }^{47}$ and Shilton et al. ${ }^{48}$ Figure S4 also provides an example of infrared images used to evaluate the temperature of the films. The results demonstrate that SAW-induced heating only affects significantly the highest viscosity used. When the viscosity of $100 \%$ glycerol was adjusted to that of the measured temperature of $50{ }^{\circ} \mathrm{C}$ (triangle in the inset), the measured relaxation time agreed well with the theoretical prediction. Hence, there is an opportunity to use this lens-free imaging system as a low-cost chip-based tool for measuring liquids viscosities. The advantage of requiring only subnanoliters sample volume cannot be underestimated for high value products, such as those used in biological experimentation.

Finally, we highlight that the deviation from this inverse proportionality of the relaxation time for $100 \%$ glycerol $(\eta=$ $612 \mathrm{cP}$ ) is due to the elastic wave heating effects (Figure 7) that have been observed for highly viscous liquids (i.e., pure glycerol). ${ }^{47,48}$ For low viscous liquids (i.e., for $\eta \leq 109 \mathrm{cP}$ ) the proposed device does not require temperature monitoring. Nonetheless, we anticipate that future versions of the device will include temperature control capability.

\section{CONCLUSIONS}

We present a method to visualize and quantitatively measure the wavelengths of guided acoustic waves using a wide field-ofview lens-free imaging system. We extend this capability to characterize beam formation and attenuation and subsequently to study the rheological properties of thin (Newtonian) liquid layers. Glycerol-water and PEG-water mixtures having different viscosities showed excellent agreement between theoretical predictions and experimental results, enabling us to establish a relationship between the transient response of the liquid-air interface and the kinematic viscosity of such liquids.

The use of ultrasmall liquid sample volumes on disposable biochips makes this technique particularly attractive for applications where rare or high value biological liquids are employed; for example, medical diagnostics or in a quality control process of pharmaceuticals. This approach could also be readily extended to non-Newtonian liquids, demonstrating the applicability of this technique in rheology.

\section{EXPERIMENTAL SECTION}

SAW Device Fabrication. Interdigitated transducers (IDT) with 40 electrode pairs $(10 \mathrm{~nm} \mathrm{Ti}, 100 \mathrm{~nm} \mathrm{Au})$ were patterned on a $128^{\circ} \mathrm{Y}$ cut $X$-propagating $\mathrm{LiNbO}_{3}$ wafer of $1 \mathrm{~mm}$ thickness (Roditi, U.K.) using standard photolithography techniques as described previously. ${ }^{49}$ Their width and pitch were designed using the simple wavelength relationship: $f=c / \lambda$ and $\lambda=D$, where $f$ is the frequency, $c$ is the wave propagation velocity of $\mathrm{LiNbO}_{3}$ and $D$ is the pitch. ${ }^{50}$ For $9.71 \mathrm{MHz}, \lambda$ was $410 \mu \mathrm{m}$. The sound propagation velocity in $\mathrm{LiNbO}_{3}$ was $3992 \mathrm{~m} /$ $\mathrm{s}$ and the IDT aperture was $20 \mathrm{~mm}$. The IDTs also showed further harmonics at 18.63 and $20.41 \mathrm{MHz}$, as measured using the $S_{11^{-}}$ parameter from a network analyzer (E5701C ENA, Agilent Technologies).

Lens-Free Computational Imaging. The lens-free microscope was developed and implemented using $3 \mathrm{D}$ printed components, as shown in Figure 1, and consisted of an illumination module, an optical band-pass filter, a sample holder attached to the SAW device and a 10 mega pixel CMOS imager (UI-1492LE-M, Imaging Development Systems). The illumination module consisted of 20 fiber coupled LEDs that were individually controlled using a microcontroller. ${ }^{51}$ Measurements were carried out using only one LED (although the presence of multiple LEDs facilitates a pixel super-resolution imaging capability $\left.^{27,28}\right)$. Imaging with a resolution below the pixel size in the future could enable studying acoustic waves at higher frequencies (shorter wavelengths).

A partially coherent light source (a fiber coupled LED, with a center wavelength of $\sim 532 \mathrm{~nm}$ ) was used to irradiate the sample. The interference between the directly transmitted and the scattered light from the sample was recorded at the CMOS imager as an inline hologram. ${ }^{52}$ The vertical distance between the sample and the CMOS detector plane was $\sim 1 \mathrm{~mm}$, such that the sample field-of-view was equal to the active area of the CMOS imager. These inline holograms could be used directly for analysis of the sample or, when spatial resolution is of importance, they can be rapidly reconstructed by digitally back-propagating the hologram to the object plane or by using iterative phase-recovery methods. ${ }^{24,53}$ Image reconstruction and elimination of twin-image related artifacts in the final holographic image were crucial in identifying smaller objects $(\sim<5 \mu \mathrm{m})$, however it was not necessary for the detection of larger spatial features of interest as presented here. Further details of this on-chip lens-free holographic imaging system can be found in the reported work. ${ }^{28}$

Samples were prepared by spin-coating the liquid of interest on the glass biochip (Fisher Finest Premium 12-548A, Fisher Scientific). The disposable glass biochips were thoroughly cleaned in acetone (67-641, Fisher) overnight and then washed with ethanol (10646134, Acros Organics) and deionized water (7732-18-5, Sigma-Aldrich). After drying, the biochips were plasma-treated (PDC-32G, Harrick Plasma) to remove any organic residuals and the sample was spin coated at $12000 \mathrm{rpm}$ for $60 \mathrm{~s}$ (WS650HZB, Laurell). The biochips were then placed on the sample holder and coupled to the SAW device using PEG400 (25322-68-3, Sigma-Aldrich). The thin coupling film had a calculated volume of about $300 \mathrm{~nL}$. The technique could also be implemented directly on the piezoelectric wafer used as a waveguide when cost and contamination issues are not critical to the target application. Wide area imaging of Lamb-type waves was performed, 
illuminated from below, with the transmitted light recorded using a CMOS imager chip.

Upon switching the ultrasonic actuation off, the acoustic wave energy at the solid-liquid boundary dissipated almost instantaneously, whereas the deformation of the liquid at the liquid-air interface relaxes at a much slower rate (over a few seconds). Rheological characterization required a volume whose geometry was defined by one acoustic wavelength $(\sim 162 \mu \mathrm{m}, x)$, averaged over 10 pixels on the CMOS sensor $(\sim 16.7 \mu \mathrm{m}, y)$, with a thickness $(z)$ of $\sim 7$ $\mu \mathrm{m}$ (i.e., $<200 \mathrm{pL}$ ). To ensure samples did not evaporate, measurements were performed in a controlled humidified environment. Throughout all experiments, sample temperature was externally monitored using a thermal imager (C2, FLIR).

In all cases, the illumination module was controlled using a LABVIEW program. The image analysis was performed using a laptop (Lenovo Y480 with an Intel Core i7-3610QM microprocessor).

Physical Characterization. Physical characterization experiments were performed as follows: The liquid sample thickness was measured on biochips by adding $20 \mu \mathrm{M}$ fluorescein dye, and a $z$-stack was obtained on a confocal microscope (LSM 510 Meta, Zeiss). Ultrasonic characterization was performed either on the $\mathrm{LiNbO}_{3}$ wafer as a Rayleigh wave, or on the glass biochip as a Lamb-type wave using a LDV (UHF-120, Polytec GmbH). An electrical network analyzer (E5701C ENA, Agilent Technologies) was used for the ultrasonic frequency characterization of the $\mathrm{LiNbO}_{3}$ transducer. Ultrasonic waves "set" in cured polymer as show in Figure 3 were measured using a scanning electron microscope (Nova 600 SEM/FIB System)

\section{ASSOCIATED CONTENT}

\section{(5 Supporting Information}

The Supporting Information is available free of charge on the ACS Publications website at DOI: 10.1021/acsnano.9b03219.

Figure S1, acoustic mode characterization; Figure S2, viscosity measurements of PEG400-water mixtures; Figure S3, change in viscosity with a $5 \mathrm{~s}$ SAW actuation (with induced heating) for glycerol-water mixtures; Figure S4, example of infrared photographs of $100 \%$ glycerol film under SAW actuation; Table S1, physical properties of materials used in numerical modeling (PDF)

Video of lens-free imaging of Lamb-type waves (MP4)

\section{AUTHOR INFORMATION}

\section{Corresponding Authors}

*E-mail: ozcan@ucla.edu. Phone: +1 (310) 5006568.

*E-mail: jon.cooper@glasgow.ac.uk. Phone: +44 (0) 141330 4931.

\section{ORCID $\odot$}

Muhammad Arslan Khalid: 0000-0002-1797-590X

Aniruddha Ray: 0000-0003-2561-2217

Manlio Tassieri: 0000-0002-6807-0385

Julien Reboud: 0000-0002-6879-8405

Aydogan Ozcan: 0000-0002-0717-683X

Jonathan M. Cooper: 0000-0002-2358-1050

\section{Present Address}

"If Aniruddha Ray: Department of Physics and Astronomy, University of Toledo, Ohio, USA.

\section{Author Contributions}

The idea was conceived by M.A.K., A.R., M.T., J.C., and A.O. The experiments and analysis were performed by M.A.K., A.R., and S.C. D.T. helped in the development of the device. M.A.K. integrated the IDT to the sample chamber. M.A.K. S.C., A.R., and A.O. developed the image analysis algorithms and the
GUI. M.A.K., A.D., J.R., and J.C. analyzed SAW propagation models. M.A.K. and M.T. performed the rheology analysis. The manuscript was written through the contributions of all the authors. J.C. and A.O. supervised the research.

\section{Author Contributions}

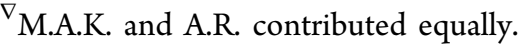

Notes

The authors declare no competing financial interest.

\section{ACKNOWLEDGMENTS}

The authors acknowledge D. Paterson (University of Glasgow) for his help with confocal microscopy, Dr. R. Wilson (University of Glasgow) for his technical support, M. Daloglu (UCLA) for his help with SEM imaging of the waves, A. Feizi (UCLA) for his help with the LabView code, M. Khan (UCLA) for his help with MATLAB codes, and the James Watt Nanofabrication Centre (University of Glasgow) for device manufacturing. J.C. acknowledges the support from EPSRC Grant (EP/K027611/1), an ERC Advanced Investigator Award (340117 Biophononics). M.K. acknowledges James Watt, David Brown Mobility, and Mac Robertson scholarships in the U.K. M.T. acknowledges support via EPSRC Grants (EP/R035067/1, EP/R035563/1, and EP/ R035156/1). A.O. acknowledges the support from Howard Hughes Medical Institute and the U.S. National Science Foundation.

\section{REFERENCES}

(1) Brillouin, L. Diffusion De La Lumière et Des Rayons X Par Un Corps Transparent Homogène - Influence De L'agitation Thermique. Ann. Phys. 1922, 9, 88-122.

(2) Raman, C.; Nagendra Nathe, N. The Diffraction of Light by High Frequency Sound Waves: Part I. Proc. - Indian Acad. Sci., Sect. A 1935, 2, 406-412.

(3) Haran, M. E. Visualization and Measurement of Ultrasonic Wavefronts. Proc. IEEE 1979, 67, 454-466.

(4) Kinsler, L. E. Fundamentals of Acoustics; John Wiley \& Sons: New York, 2000; pp 548-549.

(5) Nayfeh, A.; Nemat-Nasser, S. Thermoelastic Waves in Solids with Thermal Relaxation. Acta Mech. 1971, 12, 53-69.

(6) Mayer, G. A. Blood Viscosity and Oral Anticoagulant Therapy. Am. J. Clin. Pathol. 1976, 65, 402-406.

(7) Liu, B.; Chen, X.; Cai, H.; Ali, M. M.; Tian, X.; Tao, L.; Yang, Y.; Ren, T. Surface Acoustic Wave Devices for Sensor Applications. J. Semicond. 2016, 37, 021001-02100.

(8) Tassieri, M. Microrheology with Optaical Tweezers: Principles and Applications; Pan Stanford Publishing: Singapore, 2016; pp 219-249.

(9) Gavara, N.; Chadwick, R. Noncontact Microrheology at Acoustic Frequencies Using Frequency-Modulated Atomic Force Microscopy. Nat. Methods 2010, 7, 650-654.

(10) Horan, L. E.; Ruth, A. A.; Garcia Gunning, F. C. Hollow Core Photonic Crystal Fiber Based Viscometer with Raman Spectroscopy. J. Chem. Phys. 2012, 137, 224504.

(11) Srivastava, N.; Davenport, R. D.; Burns, M. A. Nanoliter Viscometer for Analyzing Blood Plasma and Other Liquid Samples. Anal. Chem. 2005, 77, 383-392.

(12) Lee, M.; Kim, B.; Kim, Q.; Hwang, J.; An, S.; Jhe, W. Viscometry of Single Nanoliter-Volume Droplets Using Dynamic Force Spectroscopy. Phys. Chem. Chem. Phys. 2016, 18, 2768427690.

(13) Kazys, R.; Sliteris, R.; Raisutis, R.; Zukauskas, E.; Vladisauskas, A.; Mazeika, L. Waveguide Sensor for Measurement of Viscosity of Highly Viscous Fluids. Appl. Phys. Lett. 2013, 103, 204102.

(14) Manor, O.; Rezk, A. R.; Friend, J. R.; Yeo, L. Y. Dynamics of Liquid Films Exposed to High-Frequency Surface Vibration. Phys. Rev. E 2015, 91, No. 053015. 
(15) Chen, Y.; Ding, X.; Steven Lin, S.; Yang, S.; Huang, P.; Nama, N.; Zhao, Y.; Nawaz, A.; Guo, F.; Wang, W.; Gu, Y.; Mallouk, T.; Huang, T. Tunable Nanowire Patterning Using Standing Surface Acoustic Waves. ACS Nano 2013, 7, 3306-3314.

(16) Schmid, L.; Weitz, D. A.; Franke, T. Sorting Drops and Cells with Acoustics: Acoustic Microfluidic Fluorescence-Activated Cell Sorter. Lab Chip 2014, 14, 3710-3718.

(17) Collins, D. J.; Ma, Z.; Ai, Y. Highly Localized Acoustic Streaming and Size-Selective Submicrometer Particle Concentration Using High Frequency Microscale Focused Acoustic Fields. Anal. Chem. 2016, 88, 5513-5522.

(18) Shi, J.; Ahmed, D.; Mao, X.; Lin, S.-C. S.; Lawit, A.; Huang, T. J. Acoustic Tweezers: Patterning Cells and Microparticles Using Standing Surface Acoustic Waves (SSAW). Lab Chip 2009, 9, 28902895.

(19) Neumann, T.; Ermert, H. Schlieren Visualization of Ultrasonic Wave Fields with High Spatial Resolution. Ultrasonics 2006, 44, e1561-e1566.

(20) Sapozhnikov, O. A.; Morozov, A. V.; Cathignol, D. AcoustoOptic Interaction in Laser Vibrometry in a Liquid. Acoust. Phys. 2009, 55, 365-375.

(21) Ueda, M.; Oshida, Y.; Iwata, K.; Nagata, R. Visualization of High Frequency Ultrasonic Wavefronts by Holographic Interferometry. IEEE Trans. Sonics Ultrason. 1981, 28, 436-438.

(22) Rambach, R.; Taiber, J.; Scheck, C.; Meyer, C.; Reboud, J.; Cooper, J.; Franke, T. Visualization of Surface Acoustic Waves in Thin Liquid Films. Sci. Rep. 2016, 6, 21980.

(23) Ozcan, A.; McLeod, E. Lensless Imaging and Sensing. Annu. Rev. Biomed. Eng. 2016, 18, 77-102.

(24) Greenbaum, A.; Luo, W.; Su, T.-W.; Grcs, Z.; Xue, L.; Isikman, S.; Coskun, A.; Mudanyali, O.; Ozcan, A. Imaging Without Lenses: Achievements and Remaining Challenges of Wide-Field On-Chip Microscopy. Nat. Methods 2012, 9, 889-895.

(25) Repetto, L.; Piano, E.; Pontiggia, C. Lensless Digital Holographic Microscope with Light-Emitting Diode Illumination. Opt. Lett. 2004, 29, 1132-1134.

(26) Ray, A.; Daloglu, M. U.; Ho, J.; Torres, A.; Mcleod, E.; Ozcan, A. Computational Sensing of Herpes Simplex Virus Using a CostEffective On-Chip Microscope. Sci. Rep. 2017, 7, 4856.

(27) Ray, A.; Li, S.; Segura, T.; Ozcan, A. High-Throughput Quantification of Nanoparticle Degradation Using Computational Microscopy and Its Application to Drug Delivery Nanocapsules. ACS Photonics 2017, 4, 1216-1224.

(28) McLeod, E.; Dincer, T. U.; Veli, M.; Ertas, Y. N.; Nguyen, C.; Luo, W.; Greenbaum, A.; Feizi, A.; Ozcan, A. High-Throughput and Label-Free Single Nanoparticle Sizing Based on Time-Resolved OnChip Microscopy. ACS Nano 2015, 9, 3265-3273.

(29) Issenmann, B.; Wunenburger, R.; Manneville, S.; Delville, J.-P. Bistability of a Compliant Cavity Induced by Acoustic Radiation Pressure. Phys. Rev. Lett. 2006, 97, No. 074502.

(30) Issenmann, B.; Nicolas, A.; Wunenburger, R.; Manneville, S.; Delville, J.-P. Deformation of Acoustically Transparent Fluid Interfaces by the Acoustic Radiation Pressure. Europhys. Lett. 2008, 83, 34002.

(31) Bechhoefer, J.; Ego, V.; Manneville, S.; Johnson, B. An Experimental Study of the Onset of Parametrically Pumped Surface Waves in Viscous Fluids. J. Fluid Mech. 1995, 288, 325-350.

(32) Kumar, S. Parametrically Driven Surface Waves in Viscoelastic Liquids. Phys. Fluids 1999, 11, 1970-1981.

(33) Bishara, W.; Su, T.-W.; Coskun, A. F.; Ozcan, A. Lensfree OnChip Microscopy over a Wide Field-Of-View Using Pixel SuperResolution. Opt. Express 2010, 18, 11181-11191.

(34) Nayfeh, A. H.; Nagy, P. B. Excess Attenuation of Leaky Lamb Waves Due to Viscous Fluid Loading. J. Acoust. Soc. Am. 1997, 101, 2649-2658

(35) Yapura, C. L.; Kinra, V. K. Guided Waves in a Fluid-Solid Bilayer. Wave Motion 1995, 21, 35-46.
(36) Faraday, M. On a Peculiar Class of Acoustical Figures; and on Certain Forms Assumed by Groups of Particles upon Vibrating Elastic Surfaces. Philos. Trans. R. Soc. London 1831, 121, 299-340.

(37) Chen, P.; Viñals, J. Pattern Selection in Faraday Waves. Phys. Rev. Lett. 1997, 79, 2670-2673.

(38) Kudrolli, A.; Gollub, J. P. Patterns and Spatiotemporal Chaos in Parametrically Forced Surface Waves: A Systematic Survey at Large Aspect Ratio. Phys. D 1996, 97, 133-154.

(39) Edwards, W. S.; Fauve, S. Patterns and Quasi-Patterns in the Faraday Experiment. J. Fluid Mech. 1994, 278, 123-148.

(40) Milner, S. T. Square Patterns and Secondary Instabilities in Driven Capillary Waves. J. Fluid Mech. 1991, 225, 81-100.

(41) Benjamin, T. B.; Ursell, F. J.; Taylor, G. I. The Stability of the Plane Free Surface of a Liquid in Vertical Periodic Motion. Proc. $R$ Soc., London, Ser. A 1954, 225, 505-515.

(42) Kumar, K.; Tuckerman, L. S. Parametric Instability of the Interface Between Two Fluids. J. Fluid Mech. 1994, 279, 49-68.

(43) Landau, L. D.; Lifshitz, E. M. Mechanics (Course of Theoretical Physics); Butterworth Heinemann: Singapore, 1976; Vol 1, p 169.

(44) Blamey, J.; Yeo, L. Y.; Friend, J. R. Microscale Capillary Wave Turbulence Excited by High Frequency Vibration. Langmuir 2013, 29, $3835-3845$

(45) Nazarzadeh, E.; Wilson, R.; King, X.; Reboud, J.; Tassieri, M.; Cooper, J. M. Confinement of Surface Waves at the Air-Water Interface to Control Aerosol Size and Dispersity. Phys. Fluids 2017, 29,112105

(46) Gomes, N.; Gonçalves, C.; García-Román, M.; Teixeira, J. A.; Belo, I. Optimization of a Colorimetric Assay for Yeast Lipase Activity in Complex Systems. Anal. Methods 2011, 3, 1008-1013.

(47) Zha, Y.; Zhang, A. A Microreactor Using Surface Acoustic Wave As Energy Source. 2012 Symposium on Piezoelectricity, Acoustic Waves, and Device Applications (SPAWDA). Shanghai, 2012; pp 215-218.

(48) Shilton, R. J.; Mattoli, V.; Travagliati, M.; Agostini, M.; Desii, A.; Beltram, F.; Cecchini, M. Rapid and Controllable Digital Microfluidic Heating by Surface Acoustic Waves. Adv. Funct. Mater. 2015, 25, 5895-5901.

(49) Reboud, J.; Bourquin, Y.; Wilson, R.; Pall, G.; Jiwaji, M.; Pitt, A.; Graham, A.; Waters, A.; Cooper, J. Shaping Acoustic Fields As a Toolset for Microfluidic Manipulations in Diagnostic Technologies. Proc. Natl. Acad. Sci. U. S. A. 2012, 109, 15162-15167.

(50) White, R. M.; Voltmer, F. W. Direct Piezoelectric Coupling to Surface Elastic Waves. Appl. Phys. Lett. 1965, 7, 314-316.

(51) Greenbaum, A.; Akbari, N.; Feizi, A.; Luo, W.; Ozcan, A. FieldPortable Pixel Super-Resolution Colour Microscope. PLoS One 2013, $8, \mathrm{e} 76475$.

(52) Mudanyali, O.; Tseng, D.; Oh, C.; Isikman, S. O.; Sencan, I.; Bishara, W.; Oztoprak, C.; Seo, S.; Khademhosseini, B.; Ozcan, A. Compact, Light-Weight and Cost-Effective Microscope Based on Lensless Incoherent Holography for Telemedicine Applications. Lab Chip 2010, 10, 1417-1428.

(53) Wu, Y.; Ozcan, A. Lensless Digital Holographic Microscopy and Its Applications in Biomedicine and Environmental Monitoring. Methods 2018, 136, 4-16. 